\title{
Noninvasive Ventilation
}

Editors

LISA F. WOLFE

AMEN SERGEW

\section{SLEEP MEDICINE CLINICS}

www.sleep.theclinics.com

Consulting Editor

TEOFILO LEE-CHIONG Jr

December 2020 • Volume 15 - Number 4 